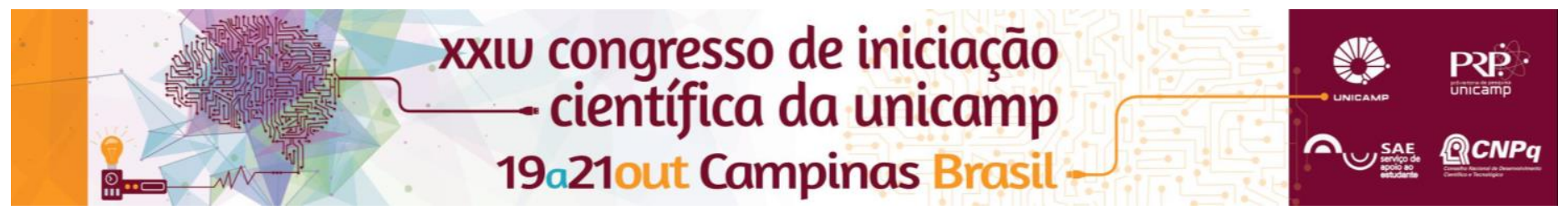

\title{
Study of Modes with Orbital Angular Momentum in Free-space, Fibers and Integrated Waveguides
}

\author{
Mateus Corato Zanarella*, Hugo Enrique Hernandez Figueroa (advisor).
}

\begin{abstract}
Modes with Orbital Angular Momentum (OAM) can be used in optical communications systems as an additional degree of freedom for the multiplexation/demultiplexation of the transmitted information, providing a substantial increase in system's capacity. The goal of this project is to study theoretically, experimentally and via computational simulations the propagation of modes with OAM in free-space, fibers and integrated silicon waveguides, taking as references the present literature and trying to make new contributions to this field of research.
\end{abstract}

\section{Key words:}

Orbital Angular Momentum, Optical Communications, Fiber Optics.

\section{Introduction}

In the last decades, many properties of the electromagnetic fields have been explored to increase the data capacity of optical links, giving rise to techniques like WDM (Wavelength Division Multiplexing) and PDM (Polarization Division Multiplexing). Recently, another technique has called attention: the SDM (Space Division Multiplexing), which uses orthogonal modes as independent channels for data transfer. Although any set of orthogonal modes can be used for this purpose, the modes with OAM are very convenient, because their circular symmetry is compatible with many commercially available optical components. The objective of this project is therefore to study theoretically, experimentally and via computational simulations the use of OAM modes in optical links, comprising free-space, special optical fibers and integrated silicon waveguides, with the goal of making new contributions to this field of research.

\section{Results and Discussion}

First, a detailed study was performed on the separation of angular momentum in orbital (OAM) and spin (SAM) parts and on the general properties of OAM modes, notably their helical phase structure and ring-shaped intensity pattern. Although this separation is, strictly speaking, only possible under paraxial regime, the same ideas are commonly applied to non-paraxial situations, but hence with an intrinsic spin-orbit coupling.

Examples of OAM beams that propagate in free-space are the Bessel beams (BB) and the Laguerre-Gauss (LB) beams. They were both experimentally generated and characterized using SLMs (Spatial Light Modulators) that operate in the visible range, available at LEMAC (Applied and Computational Electromagnetics Laboratory). Besides the qualitative characterization of single beams (by interfering them with a plane wave and counting the number of maxima on the intensity pattern), a quantitative characterization of arbitrary superpositions was made using all optical operations to perform a modal decomposition of the waves ${ }^{1}$.

In the case of optical fibers, special designs of refractive index patterns are necessary to make the propagation of OAM modes feasible. In usual fibers, the near degeneracy of the modes within the same LP group causes the ideally orthogonal OAM modes to couple under the presence of perturbations and imperfections in the fiber structure. Special refractive index profiles with a high-index ring, mimicking the intensity distribution of the modes, were then developed to lift their degeneracy and lower their coupling. The standard fibers of this type are the vortex fiber (low contrast) ${ }^{2}$ and the air-core fiber (high contrast $)^{3}$. After a modal analysis of these waveguides, we studied the coupling between adjacent modes due to bending using transformation optics ${ }^{4}$. As a new contribution, we improved the properties of these two kinds of fibers by applying a metamaterial composed of air holes around the high-index ring. This provides better confinement, permits smaller radii of curvature, reduces the coupling caused by bends and, in the air-core fiber, provides four more OAM states compared to the standard design with the same dimensions.

The experimental study of the use of OAM modes in optical fiber communications will be developed in a twomonth internship at the University of Southern California. Finally, after a study of channel rectangular silicon waveguides, we are analyzing the characteristics of the only proposal (as far as we know) of generation and propagation of OAM modes in silicon photonics 5 . Although the modes are not exactly circularly symmetric, they resemble the OAM states of a circular fiber. Some points about the robustness of the proposed method are still unclear and we are studying the possibility of making an experimental study of it using the facilities available at UNICAMP's Center of Semiconductor Components (CCS), which is something no one has done yet.

\section{Conclusions}

Although the project is still under development, we already have a novel contribution to the special optical fibers that propagate OAM modes and we expect to discover new aspects of the implementation of these modes in integrated silicon photonics.

\section{Acknowledgement}

We would like to thank FAPESP for the scientific initiation sponsorship granted to MCZ under contract No 2015/15680-2, and INCT FOTONICOM for their financial support.

\footnotetext{
${ }^{1}$ Schulze, C. et al. New J. Phys. 2013, 15, 073025.

${ }^{2}$ Ramachandran, S. et al. Opt. Lett. 2009, 34, 2525.

${ }^{3}$ Gregg, P. et al. Optica. 2015, 2, 267.

${ }^{4}$ Han, Z. et al. Opt. Lett. 2013, 38, 1778

${ }^{5}$ Zhang, D. et al. IEEE Photon. J. 2013, 5, 2201206.
} 